\title{
The Australasian Society for Infectious Diseases and Refugee Health Network of Australia recommendations for health assessment for people from refugee-like backgrounds: an abridged outline
}

\author{
Nadia J Chaves ${ }^{1,2}$, Georgia A Paxton ${ }^{3}$, Beverley-Ann Biggs ${ }^{4}$, Aesen Thambiran ${ }^{5}$, Joanne Gardiner ${ }^{6}$, Jan Williams ${ }^{7}$, \\ Mitchell M Smith ${ }^{8}$, Joshua S Davis ${ }^{9,10}$
}

$\mathrm{T}$ here are currently more than 65 million people who have been forcibly displaced worldwide, including 21.3 million people with formal refugee status, over half of whom are aged under 18 years. ${ }^{1}$ More than 15000 refugees have resettled in Australia in the 2015-16 financial year, which includes a proportion of the 12000 refugees from Syria and Iraq recently added to Australia's humanitarian intake. ${ }^{2}$ In addition, around 30000 asylum seekers who arrived by plane or boat are currently in Australia awaiting visa outcomes.

People from refugee-like backgrounds are likely to have experienced disruption of basic services, poverty, food insecurity, poor living conditions and prolonged uncertainty; they may have experienced significant human rights violations, trauma or torture. These circumstances place them at increased risk of complex physical and mental health conditions. They face numerous barriers to accessing health care after arrival in Australia, such as language, financial stress, competing priorities in the settlement period, and difficulties understanding and navigating the health care system. ${ }^{4-6}$ Most people require the assistance of an interpreter for clinical consultations. ${ }^{7}$ Offering a full health assessment to newly arrived refugees and asylum seekers is a positive step towards healthy settlement, and helps manage health inequity through the provision of catch-up immunisation and the identification and management of infectious and other health conditions.

These guidelines update the Australasian Society of Infectious Diseases (ASID) guidelines for the diagnosis, management and prevention of infectious diseases in recently arrived refugees ${ }^{8}$ published in 2009 and previously summarised in the MJA. ${ }^{9}$ When these recommendations were first published, more than $60 \%$ of humanitarian entrants arriving in Australia were from sub-Saharan Africa ${ }^{10}$ and had a high prevalence of malaria, schistosomiasis and hepatitis B virus (HBV) infection. ${ }^{11-15}$ The initial guidelines were primarily intended to help specialists and general practitioners to diagnose, manage and prevent infectious diseases. Since then, there have been changes in refugee-source countries - with more arrivals from the Middle East and Asia and fewer from sub-Saharan Africa ${ }^{16,17}$ — and an increased number of asylum seekers arriving by boat, ${ }^{18}$ alongside complex and changing asylum seeker policies and changes in health service provision for these populations. In this context, we reviewed the 2009 recommendations to ensure relevance for a broad range of health professionals and to include advice on equitable access to health care, regardless of

\section{Abstract}

Introduction: In 2009, the Australasian Society of Infectious Diseases published guidelines on the post-arrival health assessment of recently arrived refugees. Since then, the number of refugees and asylum seekers reaching Australia has increased substantially (17 555 refugees in 2015-16) and the countries of origin have changed. These groups are likely to have had poor access to health care pre-arrival and, consequently, are at risk of a range of chronic and infectious diseases. We established an advisory group that included infectious diseases physicians, general practitioners, public health specialists, paediatricians and refugee health nurses to update the 2009 guidelines.

Main recommendations: All people from refugee-like backgrounds, including children, should be offered a tailored comprehensive health assessment and management plan, ideally within 1 month of arrival in Australia. This can be offered at any time if initial contact with a GP or clinic is delayed. Recommended screening depends on history, examination and previous investigations, and is tailored based on age, gender, countries of origin and transit and risk profile. The full version of the guidelines is available at http://www.asid.net.au/ documents/item/1225.

Changes in management as a result of this guideline: These guidelines apply to all people from refugee-like backgrounds, including asylum seekers. They provide more information about non-communicable diseases and consider Asia and the Middle East as regions of origin as well as Africa. Key changes include an emphasis on person-centred care; risk-based rather than universal screening for hepatitis $C$ virus, malaria, schistosomiasis and sexually transmissible infections; updated immunisation guidelines; and new recommendations for other problems, such as nutritional deficiencies, women's health and mental health.

Medicare or visa status. The revised guidelines are intended for health care providers caring for people from refugee-like backgrounds, including GPs, refugee health nurses, refugee health specialists, infectious diseases physicians and other medical specialists.

This article summarises the full guidelines, which contain detailed literature reviews, recommendations on diagnosis and management along with explanations, supporting evidence and links to other resources. The full version is available at http://www.asid. net.au/documents/item/1225. 


\section{Methods}

The guideline development process is summarised in Box 1. The two key organisations developing these guidelines are ASID and the Refugee Health Network of Australia. ASID is Australia's peak body representing infectious diseases physicians, medical microbiologists and other experts in the fields of the prevention, diagnosis and treatment of human and animal infections. The Refugee Health Network is a multidisciplinary network of health professionals across Australia with expertise in refugee health. $^{20}$

We defined clinical questions using the PIPOH framework (population, intervention, professionals, outcomes and health care setting). ${ }^{21}$ The chapter authors and the Expert Advisory Group developed recommendations based on reviews of available evidence, using systematic reviews where possible. Australian prevalence data also informed screening recommendations; for example, the low reported prevalence of chlamydia $(0.8-2.0 \%)$ infections and absence of gonorrhoea infections in refugee cohorts in Australia ${ }^{13,22-24}$ (and in other developed countries ${ }^{25-27}$ ) informed the new recommendation for risk-based sexually transmitted infection (STI) screening.

Despite the intention to assign levels of evidence to each recommendation, there was limited published high level evidence in most areas, and virtually all recommendations are based on

\section{Guideline development process}

- An EAG, consisting of refugee health professionals, was formed and it included two ID physicians, an ID and general physician, two GPs, a public health physician, a general paediatrician and a refugee health nurse. An editorial subgroup was also formed.

- The EAG determined the list of priority conditions in consultation with refugee health specialists and RACGP Refugee Health Special Interest Group clinicians, incorporating information from consultations with refugee background communities ${ }^{19}$ and previous ASID refugee health guidelines.

- Each condition was assigned to a primary specialist author with paediatrician and primary care or specialist co-authors. Twenty-eight authors from six states and territories were involved in writing the first draft.

- The EAG reviewed the first draft to ensure consistency with the framework and the rest of the guidelines. They were then revised by the primary authors.

- External expert review authors reviewed the second draft and they were then revised by the primary authors.

- The EAG and the refugee health nurse subcommittee reviewed the third draft.

- The stakeholders reviewed the fourth draft: ASID, NTAC, RHeaNA, RACGP Refugee Health Special Interest Group, RACP, RACP AChSHM, the Victorian Foundation for the Survivors of Torture, the Multicultural Centre for Women's Health, the Asylum Seeker Resource Centre, the Ethnic Communities Council of Victoria and community members.

- The comments from the stakeholders were returned to the authors for review and the EAG compiled the final version.

- $A S I D, R A C P, N T A C$ and AChSHM endorsed the final version.

AChSHM = Australasian Chapter of Sexual Health Medicine. ASID = Australasian Society for Infectious Diseases. EAG = Expert Advisory Group. GP = general practitioner. ID = infectious diseases. NTAC = National Tuberculosis Advisory Council. RACGP = Royal Australian College of General Practitioners. RACP $=$ Royal Australasian College of Physicians. RHeaNA = Refugee Health Network of Australia. Adapted from the ASID and RHeaNA Recommendations for comprehensive post-arrival health assessment for people from refugee-like backgrounds (2016: https://www.asid.net.au/documents/item/1225) with permission from ASID. expert consensus. Consensus was not reached regarding the recommendations relating to human immunodeficiency virus (HIV) and STIs.

The term "refugee-like" is used to describe people who are refugees under the United Nations Refugee Convention, ${ }^{28}$ those who hold a humanitarian visa, people from refugee-like backgrounds who have entered under other migration streams, and people seeking asylum in Australia. "Refugee-like" acknowledges that people may have had refugee experience in their countries of origin or transit, but do not have formal refugee status.

\section{Current pre-departure screening}

All permanent migrants to Australia have a pre-migration immigration medical examination 3-12 months before departure, ${ }^{29}$ which includes a full medical history and examination. Investigations depend on age, risk factors and visa type, ${ }^{30}$ and include:

- a chest $x$-ray for current or previous tuberculosis ([TB]; age $\geq 11$ years);

- screening for latent TB infection with an interferon- $\gamma$ release assay or tuberculin skin test (for children aged 2-10 years, if they hold humanitarian visas, come from high prevalence countries or have had prior household contact);

- HIV serology (age $\geq 15$ years, unaccompanied minors);

- hepatitis B surface antigen (HBsAg) testing (pregnant women, unaccompanied minors, onshore protection visas, health care workers);

- hepatitis $\mathrm{C}$ virus (HCV) antibody testing (onshore protection visas, health care workers); and

- $\quad$ syphilis serology (age $\geq 15$ years, humanitarian visas, onshore protection visas).

Humanitarian entrants are also offered a voluntary pre-departure health check depending on departure location and visa subtype. $^{31}$ The pre-departure health check includes a rapid diagnostic test and treatment for malaria in endemic areas; empirical treatment for helminth infections with a single dose of albendazole; measles, mumps and rubella vaccination; and yellow fever and polio vaccination where relevant. The current cohort of refugees arriving from Syria will have extended screening incorporating the immigration medical examination and pre-departure health check, with additional mental health review and immunisations.

People seeking asylum who arrived by boat have generally had a health assessment on arrival in immigration detention - although clinical experience suggests that investigations and detention health care varies, especially for children. However, asylum seekers who arrived by plane will not have had a pre-departure immigration medical examination.

\section{General recommendations}

Our overarching recommendation is to offer all people from refugee-like backgrounds, including children, a comprehensive health assessment and management plan, ideally within 1 month of arrival in Australia. This assessment can be offered at any time after arrival if the initial contact with a GP or clinic is delayed, and should also be offered to asylum seekers after release from detention. Humanitarian entrants who have been in Australia for less than 12 months are eligible for a GP Medicare-rebatable health 
assessment. Such assessments may take place in a primary care setting or in a multidisciplinary refugee health clinic. Documented overseas screening and immunisations, and clinical assessment should also guide diagnostic testing.

Health care providers should adhere to the principles of personcentred care when completing post-arrival assessments. ${ }^{32,33}$ These include: respect for the patient's values, preferences and needs; coordination and integration of care with the patient's family and other health care providers; optimising communication and education, provision of interpreters where required (the Doctors Priority Line for the federal governmentfunded Translating and Interpreting Service is 1300131 450) and use of visual and written aids and teach-back techniques to support health literacy. ${ }^{34}$ It is important to explain that a health assessment is voluntary and results will not affect visa status or asylum claims.

\section{Specific recommendations}

Recommendations are divided into two sections: infectious and non-infectious conditions. Box 2 provides a checklist of all recommended tests, and Box 3 sets out details of country-specific recommendations. A brief overview is provided below. For more detailed recommendations regarding management, follow-up and considerations for children and in pregnancy, see the full guidelines.

\section{Short checklist of recommendations for post-arrival health assessment of people from refugee-like backgrounds \\ Offer test to \\ All Test \\ Full blood examination \\ Hepatitis B serology (HBsAg, HBsAb, HBcAb) \\ Strongyloides stercoralis serology HIV serology* \\ TST or IGRA \\ Varicella serology \\ Visual acuity \\ Glaucoma assessment \\ Dental review \\ Hearing review \\ Social and emotional wellbeing and mental health \\ Developmental delay or learning concerns \\ Preventive health as per RACGP ${ }^{35}$ \\ Catch-up vaccinations \\ Risk-based \\ Rubella IgG \\ Ferritin \\ Vitamin D, also check calcium, phosphate, and alkaline phosphatase in children \\ Vitamin $\mathrm{B}_{12}$ \\ First pass urine or self-obtained vaginal swabs for gonorrhoea and chlamydia PCR \\ Syphilis serology \\ Helicobacter pylori stool antigen or breath test \\ Stool microscopy (ova, cysts and parasites) \\ Country-based (Box 3) \\ Schistosoma serology \\ Malaria thick and thin films and rapid diagnostic test HCV $A b$, and HCV RNA if HCV Ab positive

Comments and target condition \\ Anaemia, iron deficiency, eosinophilia \\ HBsAg testing introduced overseas in 2016 for Syrian and Iraqi refugee cohort and may have been completed in other groups \\ Strongyloidiasis \\ $\geq 15$ years or unaccompanied or separated minor \\ Also part of IME for age $\geq 15$ years \\ Offer test if intention to treat. All $\leq 35$ years; if \\ $\geq 35$ years, depends on risk factors and local jurisdiction. TST preferred for children $<5$ years TST or IGRA testing introduced in 2016 as part of IME for children 2-10 years (humanitarian entrants, high prevalence countries, prior household contact) LTBI \\ $\geq 14$ years if no known history of disease \\ Determine immunisation status \\ Vision status, other eye disease \\ Africans $>40$ years and others $>50$ years \\ Caries, periodontal disease, other oral health issues Hearing impairment \\ Mental illness, trauma exposure, protective factors \\ Children and adolescents \\ Developmental issues, disability, trauma exposure \\ Non-communicable diseases, consider screening earlier than usual age \\ Vaccine preventable diseases, including hepatitis B \\ Women of childbearing age \\ Determines immunisation status \\ Men who have risk factors, women and children Iron deficiency anaemia \\ Risk factors if dark skin or lack of sun exposure Low vitamin $D$, rickets \\ Arrival $<6$ months, food insecurity, vegan diet or from Bhutan, Afghanistan, Iran or Horn of Africa Nutritional deficiency, risk for developmental disability in infants \\ Risk factors for STI or on request* \\ Risk factors for STIs, unaccompanied or separated minors. Part of IME in humanitarian entrants aged $\geq 15$ years \\ Gastritis, peptic ulcer disease, family history of gastric cancer, dyspepsia \\ If no documented pre-departure albendazole or persisting eosinophilia despite albendazole Intestinal parasites \\ Schistosomiasis \\ Malaria \\ $\mathrm{HCV}$, also test if risk factors, regardless of country of origin}

$\mathrm{HBCAb}=$ hepatitis B core antibody. HBsAb = hepatitis B surface antibody. HBsAg = hepatitis B surface antigen. $\mathrm{HCV}=$ hepatitis $\mathrm{C}$ virus. HCV Ab = hepatitis C antibody.

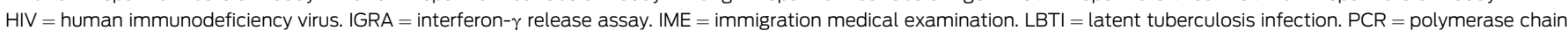
reaction. TST $=$ tuberculin skin test. * The panel did not reach consensus on these recommendations. See full guideline at http://www.asid.net.au/documents/item/1225 for details. 
3 Top 20 countries of origin for refugees and asylum seekers ${ }^{2,3,16}$ and country-specific recommendations for malaria, schistosomiasis and hepatitis C screening*

\begin{tabular}{|c|c|c|c|}
\hline Country of birth & Malaria $^{36}$ & Schistosomiasis $^{37}$ & Hepatitis $\mathrm{C}^{\dagger 38}$ \\
\hline Afghanistan & No & No & No \\
\hline Bangladesh & Yes & No & No \\
\hline Bhutan & Yes & No & No \\
\hline Burma & Yes & Yes & No \\
\hline China & No & No & No \\
\hline Congo & Yes & Yes & Yes \\
\hline Egypt & No & Yes & Yes \\
\hline Eritrea & Yes & Yes & No \\
\hline India & Yes & Yes & No \\
\hline Iran & No & No & No \\
\hline Iraq & No & Yes & Yes \\
\hline Lebanon & No & No & No \\
\hline Pakistan & Yes & No & Yes \\
\hline Somalia & Yes & Yes & No \\
\hline Sri Lanka & Yes & No & No \\
\hline Stateless ${ }^{\ddagger}$ & Yes & Yes & No \\
\hline Sudan & Yes & Yes & No \\
\hline Syria & No & Yes & Consider \\
\hline Vietnam & No & No & No \\
\hline
\end{tabular}

* There are regional variations in the prevalence of these conditions within some countries. We have taken the conservative approach of recommending screening for all people from an endemic country rather than basing the recommendation on exact place of residence. Note that some refugees and asylum seekers may have been exposed during transit through countries not listed here. See full guideline for further details. $†$ People with risk factors for hepatitis $C$ should be tested regardless of country of origin. $\ddagger$ "Stateless" in this table refers to people of Rohingyan origin Adapted from the ASID and RHeaNA Recommendations for comprehensive post-arrival health assessment for people from refugee-like backgrounds (2016; https://www.asid.net.au/documents/item/1225) with permission from ASID.

\section{Infectious conditions}

TB:

- Offer latent TB infection testing with the intention to offer preventive treatment and follow-up.

- Offer screening for latent TB infection to all people aged $\leq 35$ years.

- Children aged 2-10 years may have been screened for latent TB infection as part of their pre-departure screening.

- Screening and preventive treatment for latent TB infection in people $>35$ years will depend on individual risk factors and jurisdictional requirements in the particular state or territory.

- Use either a tuberculin skin test or interferon- $\gamma$ release assay (blood) to screen for latent TB infection.

- A tuberculin skin test is preferred over interferon- $\gamma$ release assay for children $<5$ years of age.

- Refer patients with positive tuberculin skin test or interferon- $\gamma$ release assay results to specialist tuberculosis services for assessment and exclusion of active TB and consideration of treatment for latent TB infection.

- Refer any individuals with suspected active TB to specialist services, regardless of screening test results.
Malaria:

- Investigations for malaria should be performed for anyone who has travelled from or through an endemic malaria area (Box 3), within 3 months of arrival if asymptomatic, or any time in the first 12 months if there is fever (regardless of pre-departure malaria testing or treatment).

- Test with both thick and thin blood films and an antigenbased rapid diagnostic test.

- All people with malaria should be treated by, or in consultation with, a specialist infectious diseases service.

HIV:

- Offer HIV testing to all people aged $\geq 15$ years and all unaccompanied or separated minors, as prior negative tests do not exclude the possibility of subsequent acquisition of HIV (note that consensus was not reached regarding this recommendation).

HBV:

- Offer testing for HBV infection to all, unless it has been completed as part of the immigration medical examination.

- A complete HBV assessment includes HBsAg, HB surface antibody and HB core antibody testing.

- If the HBsAg test result is positive, further assessment and follow-up with clinical assessment, abdominal ultrasound and blood tests are required.

$\mathrm{HCV}$ :

- Offer testing for HCV to people if they have:

- risk factors for $\mathrm{HCV}$;

- lived in a country with a high prevalence $(>3 \%)$ of $\mathrm{HCV}$ (Box 3); or

- an uncertain history of travel or risk factors.

- Initial testing is with an HCV antibody test. If the result is positive, request an HCV RNA test.

- If the HCV RNA test result is positive, refer to a doctor accredited to treat HCV for further assessment.

Schistosomiasis:

- Offer blood testing for Schistosoma serology if people have lived in or travelled through endemic countries (Box 3).

- If serology is negative, no follow-up is required.

- If serology is positive or equivocal:

- treat with praziquantel in two doses of $20 \mathrm{mg} / \mathrm{kg}$, 4 hours apart, orally; and

- perform stool microscopy for ova, urine dipstick for haematuria, and end-urine microscopy for ova if there is haematuria.

- If ova are seen in urine or stool, evaluate further for end-organ disease.

Strongyloidiasis:

- Offer blood testing for Strongyloides stercoralis serology to all.

- If serology is positive or equivocal:

- check for eosinophilia and perform stool microscopy for ova, cysts and parasites; and

- treat with ivermectin $200 \mu \mathrm{g} / \mathrm{kg}$ (weight $\geq 15 \mathrm{~kg}$ ), on days 1 and 14 and repeat eosinophil count and stool sample if abnormal. 
- Refer pregnant women or children $<15 \mathrm{~kg}$ for specialist management.

Intestinal parasites:

- Check full blood examination for eosinophilia.

- If pre-departure albendazole therapy is documented:

- if there are no eosinophilia and no symptoms, no investigation or treatment is required; and

- if there is eosinophilia, perform stool microscopy for ova, cysts and parasites, followed by directed treatment.

- If no documented pre-departure albendazole therapy, depending on local resources and practices, there are two acceptable options:

- empirical single dose albendazole therapy (age $>6$ months, weight $<10 \mathrm{~kg}$, dose $200 \mathrm{mg}$; weight $\geq 10 \mathrm{~kg}$, dose $400 \mathrm{mg}$; avoid in pregnancy, class D drug); or

- perform stool microscopy for ova, cysts and parasites, followed by directed treatment.

Helicobacter pylori:

- Routine screening for $H$. pylori infection is not recommended.

- Screen with either stool antigen or breath test in adults from high risk groups (family history of gastric cancer, symptoms and signs of peptic ulcer disease, or dyspepsia).

- Children with chronic abdominal pain or anorexia should have other common causes of their symptoms considered in addition to $H$. pylori infection.

- Treat all those with a positive test (see the full guidelines for details, tables 1.5 and 9.1).

STIs:

- Offer an STI screen to people with a risk factor for acquiring an STI or on request. Universal post-arrival screening for STIs for people from refugee-like backgrounds is not supported by current evidence.

- A complete STI screen includes a self-collected vaginal swab or first pass urine nucleic acid amplification test and consideration of throat and rectal swabs for chlamydia and gonorrhoea, and serology for syphilis, HIV and HBV.

- Syphilis serology should be offered to unaccompanied and separated children $<15$ years.

Skin conditions:

- The skin should be examined as part of the initial physical examination.

- Differential diagnoses will depend on the area of origin (see table 11.1 in full guidelines for details).

Immunisation:

- Provide catch-up immunisation so that people of refugee background are immunised equivalent to an Australian-born person of the same age.

- In the absence of written immunisation documentation, full catch-up immunisation is recommended.

- Varicella serology is recommended for people aged $\geq 14$ years if there is no history of natural infection.

- Rubella serology should be completed in women of childbearing age.

\section{Non-infectious conditions}

Anaemia and other nutritional problems:

- Offer full blood examination screening for anaemia and other blood conditions to all.

- Offer screening for iron deficiency with serum ferritin to children, women of childbearing age, and men who have risk factors.

- Check vitamin D status as part of initial health screening in people with one or more risk factors for low vitamin D.

- People with low vitamin D should be treated to restore their levels to the normal range with either daily dosing or high dose therapy, paired with advice about sun exposure.

- Consider screening for vitamin $\mathrm{B}_{12}$ deficiency in people with history of restricted food access, especially those from Bhutan, Afghanistan, Iran and the Horn of Africa.

Chronic non-communicable diseases in adults:

- Offer screening for non-communicable diseases in line with the Royal Australian College of General Practitioners Red Book $^{35}$ recommendations, including assessment for:

- smoking, nutrition, alcohol and physical activity;

- obesity, diabetes, hypertension, cardiovascular disease, chronic obstructive pulmonary disease and lipid disorders; and

- breast, bowel and cervical cancer.

- Assess diabetes and cardiovascular disease risk earlier for those from regions with a higher prevalence of noncommunicable diseases, or those with an increased body mass index or waist circumference.

Mental health:

- A trauma informed assessment of emotional wellbeing and mental health is part of post-arrival screening. Being aware of the potential for past trauma and impact on wellbeing is essential, although it is generally not advisable to ask specifically about details in the first visits.

- Consider functional impairment, behavioural difficulties and developmental progress as well as mental health symptoms when assessing children.

Hearing, vision and oral health:

- A clinical assessment of hearing, visual acuity and dental health should be part of primary care health screening.

Women's health:

- Offer women standard preventive screening, taking into account individual risk factors for chronic diseases and bowel, breast and cervical cancer.

- Consider pregnancy and breastfeeding and offer appropriate life stage advice and education, such as contraceptive advice where needed, to all women, including adolescents.

- Practitioners should be aware of clinical problems, terminology and legislation related to female genital mutilation or cutting and forced marriage.

Acknowledgements: We thank the following people for their input to the full version of the guideline: chapter authors Marion Bailes, Sarah Cherian, Vanessa Clifford, Benjamin Cowie, Justin Denholm, Rebecca Dunn, Josh Francis, Debbie Hocking, David Isaacs, Margaret Kay, Karen Kiang, Chris Lemoh, Jennifer Maclachlan, Christine Phillips, Sahema Saberi, Thomas Schulz, Gillian Singleton, Kasha Singh, 
Kate Walker and Shanti Narayanasamy; expert reviewers John Furler, Peter Greenberg, Karin Leder, James McCarthy, Ric Price, Regina Quiazon, Nathan Ryder, Harsha Sheorey, Rebecca Szabo, Alfred Chin Yen Tay, Justin Waring and Mary Webberley; Refugee Health Nurse Subcommittee members Jan Williams, Merilyn Spratling, Sandy Eagar and Lindy Marlow; proofreaders and editors Linny Kimly Phuong, Shanti Narayanasamy, Kudzai Kanhutu and Deborah Norberry.

Competing interests: Nadia Chaves received funding from the Victorian Department of Health and Human Services to coordinate the production of this resource as the 2015 Royal Melbourne Hospital
Refugee Health Fellow based at the Peter Doherty Institute for Infection and Immunity. Joanne Gardiner, Thomas Schulz and Karen Kiang were also funded as Victorian Refugee Health Fellows. Joshua Davis holds a career development fellowship of the National Health and Medical Research Council (1083105).

Provenance: Not commissioned; not externally peer reviewed.

C 2017 AMPCo Pty Ltd. Produced with Elsevier B.V. All rights reserved.
1 United Nations High Commission for Refugees. Global trends: forced displacement in 2015. Geneva: UNHCR: 2016. http://www.unhcr.org/576408cd7.pdf (accessed Sept 2016).

2 Department of Immigration and Border Protection. 2015-16 Humanitarian programme outcomes. Canberra: Commonwealth of Australia; 2016. http://www.border. gov.au/ReportsandPublications/Documents/statistics/ humanitarian-programme-outcomes-offshore-2015-16. pdf (accessed March 2017).

3 Department of Immigration and Border Protection. IMA legacy caseload report on status and processing outcomes. Canberra: Commonwealth of Australia; 2016 http://www.border.gov.au/ReportsandPublications/ Documents/statistics/ima-legacy-caseload-dec-16.pdf (accessed Mar 2017).

4 Joshi C, Russell G, Cheng I-H, et al. A narrative synthesis of the impact of primary health care delivery models for refugees in resettlement countries on access, quality and coordination. Int J Equity Health 2013; 12: 88.

5 Kulkens M. Access to specialist services by refugees in Victoria: A report prepared for the Department of Human Services by the Victorian Refugee Health Network. Brunswick: Victorian Refugee Health Network; 2009. http://refugeehealthnetwork.org.au/wp-content/ uploads/Specialist_access_reportNov301.pdf (accessed Sept 2016).

6 Spike EA, Smith MM, Harris MF. Access to primary health care services by community-based asylum seekers. Med J Aust 2011; 195: 188-191. https://www.mja.com.au/ journal/2011/195/4/access-primary-health-care-servicescommunity-based-asylum-seekers

7 Victorian Foundation for the Survivors of Torture. Promoting the engagement of interpreters in Victorian health services. Brunswick: Victorian Foundation for the Survivors of Torture; 2013. http://refugeehealthnetwork. org.au/wp-content/uploads/FHinterp_June2013_for web.pdf (accessed Sept 2016).

8 Murray R, Davis JS, Krause VL, et al. Diagnosis, management and prevention of infections in recently arrived refugees. Sydney: Australasian Society for Infectious Diseases; 2009.

9 Murray RJ, Davis JS, Burgner DP. The Australasian Society for Infectious Diseases guidelines for the diagnosis, management and prevention of infections in recently arrived refugees: an abridged outline. Med J Aust 2009; 190: 421-425. https://www.mja.com.au/ journal/2009/190/8/australasian-society-infectiousdiseases-guidelines-diagnosis-management-and

10 Department of Immigration and Citizenship. Annual report 2006-07. Canberra: Commonwealth of Australia; 2007. https://www.border.gov.au/ReportsandPublications/ Documents/annual-reports/annual-report-2006-07complete.pdf (accessed Feb 2017).

11 Martin JA, Mak DB. Changing faces: a review of infectious disease screening of refugees by the Migrant Health Unit, Western Australia in 2003 and 2004. Med J Aust 2006; 185: 607-610. https://www.mja.com.au/ journal/2006/185/11/changing-faces-review-infectiousdisease-screening-refugees-migrant-health-unit

12 Trauer JM, Krause VL. Assessment and management of latent tuberculosis infection in a refugee population in the Northern Territory. Med J Aust 2011; 194: 579-582 https://www.mja.com.au/journal/2011/194/11/ assessment-and-management-latent-tuberculosisinfection-refugee-population

13 Gibney KB, Mihrshahi S, Torresi J, et al. The profile of health problems in African immigrants attending an infectious disease unit in Melbourne, Australia. Am J Trop Med Hyg 2009; 80: 805-811.

14 Sheikh M, Pal A, Wang S, et al. The epidemiology of health conditions of newly arrived refugee children: $A$ review of patients attending a specialist health clinic in Sydney. J Paediatr Child Health 2009; 45: 509-513.

15 Johnston V, Smith L, Roydhouse H. The health of newly arrived refugees to the Top End of Australia: results of a clinical audit at the Darwin Refugee Health Service. Aust J Prim Health 2012; 18: 242-247. http://www.publish. csiro.au/PY/PY11065

16 Department of Immigration and Border Protection Australia's humanitarian programme - 2013-14 Canberra: Commonwealth of Australia; 2015. https:// www.border.gov.au/ReportsandPublications/ Documents/statistics/humanitarian-statistics-2013-14. pdf\#search=humanitarian statistics (accessed Sept 2016).

17 Department of Immigration and Border Protection. Australia's offshore humanitarian programme: 2013-14. Canberra: Commonwealth of Australia, 2014. https:// www.border.gov.au/ReportsandPublications/ Documents/statistics/australia-offshore-humanitarianprogram-2013-14.pdf (accessed Sept 2016).

18 Department of Immigration and Border Protection Asylum trends Australia: 2012-13, annual publication. Canberra: Commonwealth of Australia; 2012. https:// www.border.gov.au/ReportsandPublications/ Documents/statistics/asylum-trends-aus-2012-13.pdf (accessed Sept 2016).

19 Department of Health and Human Services, State Government of Victoria. Victorian refugee health and wellbeing action plan: consultation summary. Melbourne: State of Victoria; 2013. https://www2.health vic.gov.au/getfile/?sc_itemid=\%7BA6E3FC63-A4AB-42 B6-9C15-A23518737ADB\%7D\&title=Consultation \% 20Summary\%20-\%20Victorian\%20refugee\%20health $\% 20$ and\%20wellbeing\%20action\%20plan (accessed Sept 2016)

20 Phillips CB, Smith MM, Kay M, Casey S. The Refugee Health Network of Australia: towards national collaboration on health care for refugees. Med J Aust 2011; 195: 185-186. https://www.mja.com.au/journal/ 2011/195/4/refugee-health-network-australia-towardsnational-collaboration-health-care

21 ADAPTE Collaboration. Guideline adaptation: a resource toolkit. 2nd ed. ADAPTE Collaboration; 2009. http:// www.g-i-n.net/document-store/working-groupsdocuments/adaptation/adapte-resource-toolkitguideline-adaptation-2-0.pdf (accessed Sept 2016).

22 Paxton GA, Sangster KJ, Maxwell EL, et al. Post-arrival health screening in Karen refugees in Australia. PLoS One 2012; 7: e38194.

23 Chaves NJ, Gibney KB, Leder K, et al. Screening practices for infectious diseases among Burmese refugees in Australia. Emerg Infect Dis 2009; 15: 1769-1772.

24 Hoad VC, Thambiran A. Evaluating the chlamydia and gonorrhoea screening program in the Humanitarian Entrant Health Service, Western Australia. Med J Aust
2012; 197: 47-49. https://www.mja.com.au/journal/ 2012/197/1/evaluating-chlamydia-and-gonorrhoeascreening-program-humanitarian-entrant-health

25 Stauffer WM, Painter J, Mamo B, et al. Sexually transmitted infections in newly arrived refugees: is routine screening for Neisseria gonorrheae and Chlamydia trachomatis infection indicated? Am J Trop Med Hyg 2012; 86: 292-295.

26 Centers for Disease Control and Prevention. Refugee health guidelines: screening for sexually transmitted diseases during the domestic medical examination for newly arrived refugees. Atlanta: CDC; 2014. http://www. cdc.gov/immigrantrefugeehealth/guidelines/domestic/ sexually-transmitted-diseases/index.html (accessed Sept 2016).

27 Redditt VJ, Janakiram P, Graziano D, Rashid M. Health status of newly arrived refugees in Toronto, Ont: part 1: infectious diseases. Can Fam Physician 2015; 61: e303-e309.

28 UNHCR. The 1951 convention and its 1967 protocol. Geneva; UHNCR2011. http://www.unhcr.org/4ec262df9. html (accessed Sept 2016).

29 Department of Immigration and Border Protection. Panel member instructions: Australian immigration medical examinations, July 2016. Canberra: Commonwealth of Australia; 2016. https://www.border. gov.au/Panelphysicians/Documents/panel-memberinstructions.pdf (accessed Sept 2016).

30 Department of Immigration and Border Protection. Health examinations. Canberra: Commonwealth of Australia; 2015. http://www.border.gov.au/Trav/Visa/ Heal/meeting-the-health-requirement/healthexaminations (accessed Sept 2016).

31 Department of Immigration and Border Protection. Fact sheet: departure health check. Canberra:

Commonwealth of Australia; 2016. https://www.border. gov.au/about/corporate/information/fact-sheets/67adhc (accessed Sept 2016).

32 Australian Commission on Safety and Quality in Health Care. Patient-centred care: improving quality and safety through partnerships with patients and consumers. Sydney: ACSQHC; 2011. http://www.safetyandquality. gov.au/wp-content/uploads/2012/03/PCC_Paper_ August.pdf (accessed Sept 2016).

33 Picker Institute. Picker principles of patient-centred care. http://cgp.pickerinstitute.org/?page_id=1319 (accessed Sept 2016).

34 Batterham RW, Hawkins M, Collins PA, et al. Health literacy: applying current concepts to improve health services and reduce health inequalities. Public Health 2016; 132: 3-12

35 Royal Australian College of General Practitioners. Guidelines for preventive activities in general practice. 9th ed. Melbourne: RACGP; 2016. http://www.racgp.org.au/ your-practice/guidelines/redbook (accessed Sept 2016).

36 World Health Organization. International travel and health: malaria update 2015. Geneva: WHO; 2015. http://www.who. int/ith/2015-ith-chapter7.pdf?ua=1 (accessed Sept 2016).

37 World Health Organization. Status of schistosomiasis endemic countries 2015. Geneva: WHO; 2015. http:/ apps.who.int/neglected_diseases/ntddata/sch/sch.html (accessed Sept 2016).

38 Lavanchy D. Evolving epidemiology of hepatitis C virus. Clin Microbiol Infect 2011; 17: 107-115. 